\title{
Next-generation Alzheimer's Therapeutics: Leveraging Deep Biology
}

\author{
F.M. Longo' S.M. Massa²

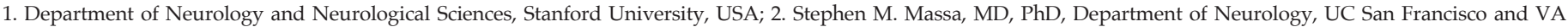 \\ Health Care System, San Francisco, USA \\ Correspondance author: Frank M. Longo, Department of Neurology and Neurological Sciences, Stanford University, USA, flongo@stanford.edu \\ J Prev Alz Dis 2020;3(7):138-139 \\ Published online May 13, 2020, http:/ / dx.doi.org/10.14283/jpad.2020.30
}

A recent EU/US CTAD Task Force Report focused on non-amyloid approaches to Alzheimer's disease (AD) modification (1). While the broad range of targets and therapies highlighted is in some ways sobering, several themes and advances in the field point to principles and technologies that are encouraging and will likely accelerate progress. These themes include: the view that amyloid and nonamyloid approaches might ultimately be complementary or synergistic; the biological diversity of approaches; emerging -omics strategies that might help guide such options; and finally, the incorporation of aging biology into perspectives of target prioritization and disease modification.

Given the swings between discouragement and promise in amyloid-based therapy trials (2), most recently with data regarding the EMERGE and ENGAGE trials in early-stage $\mathrm{AD}$ and the DIAN-TU study in asymptomatic at-risk subjects, it is notable that the task force report captures the view that non-amyloid approaches are not necessarily replacements for 'unsuccessful' approaches but are avenues to potential co-therapies. Indeed, a number of alternative approaches can be viewed from the perspective of being up- or down-stream of core amyloid mechanisms, or alternatively, constituting parallel strategies to mitigate primary neurodegenerative mechanisms with or without known links to amyloid. Interestingly, evidence suggests that degenerative tau mechanisms might occur independently of amyloid beta $(\mathrm{A} \beta)$ and yet be promoted by $\mathrm{AD}$ genetic risk factors (3). There is ample evidence indicating that processes involving $\mathrm{A} \beta$, tau, inflammation and other factors likely interact in fundamental ways to promote AD (4). Challenges intrinsic to tau-based approaches include the many potential distinct strains/isoforms of tau, and in the case of tau-lowering efforts, the feasibility of chronic tau reduction in the context of its physiological functions. Anti-inflammation approaches will need to distinguish and address the beneficial or physiological roles of the immune response, such as clearance of $A \beta$, versus disease-causing toxic mechanisms.

Data available so far from amyloid-based presymptomatic and early-stage $\mathrm{AD}$ trials indicates that even with successful trial outcomes, the role for non-amyloid approaches will be vital. With a life expectancy of some 25 or more years for a middle-aged, pre-symptomatic subject, a delay of disease onset by a few years would constitute an important breakthrough, but would leave a critical need for a therapeutic strategy that could delay onset in an even more powerful way. Similarly, for earlystage patients, a reduction in the downward slope of cognitive decline would again be a major step forward, but would fall short of the goal of the development of interventions that can truly halt progression, or better, to reverse the trajectory of synaptic failure, as has been achieved in some mouse studies.

The CTAD Task Force reviewed and advocated for a biologically diverse collection of approaches including those inhibiting tau- and inflammation-mediated degeneration and those focused on neuroprotection, growth factor mechanisms, metabolic effects, neurostimulation and lifestyle interventions. This diversity of approaches is likely only the beginning of the emergence of a vast number of therapeutic targets and strategies. Genome-wide association and transcriptome studies and work integrating proteome and other systems biology approaches (5) continue to identify numerous important new candidates. Insights from improved methods for the identification of protein-protein interaction networks and protein hubs and bottlenecks are of particular interest $(6,7)$. A number of non-amyloid approaches, including those reducing tau levels or modulating neurotrophin receptor signaling, demonstrate the proof-of-concept that multiple fundamental mechanisms, such as those regulating inflammation or synaptic integrity, can be engaged by single therapies. Thus, the development of targeted therapies that affect in parallel, several, largely distinct basic biological processes that underlie degeneration is an attractive possibility, especially given the difficulties in executing combinatoric clinical trials.

While the abundance of target candidates is a positive development for the $\mathrm{AD}$ field, it presents the challenge of prioritizing approaches both in terms of likely intrinsic efficacy and the potential to be synergistic with amyloidbased approaches. Fortunately, newly established systems biology capabilities and collaborative teams are addressing this issue. Advances in integrating 
systems biology data science, the ever-increasing rich array of human molecular and biomarker data (such as that made available by Religious Orders Study and the Rush Memory and Aging Project), and increasing sophistication in merging and analyses of human and animal model-based databases, are creating new ways to identify and prioritize target modules. Moreover, recently created consortia such as AMP-AD and TREAT$\mathrm{AD}$ funded by the National Institute of Aging are bringing teams together with a remarkable spectrum of expertise ranging from mathematical modeling to data science, biology and drug development for the purpose of identifying and facilitating the exploration of entirely new generations of candidate treatments.

Fitting quite well with the plethora of emerging candidate target mechanisms are expanding options in biomarker approaches (8). New biomarkers and advances in biomarker assessment will make smaller-scale and lower-cost exploratory endpoint trials with better defined and selected subject populations more feasible, allowing us to better leverage the increasing number of candidate therapeutic strategies. Moreover, systems biology studies will likely offer additional novel biomarkers.

Another important theme is the acknowledgment that late-onset AD is highly age-dependent and that elucidating aging biology, and in particular links to underlying AD mechanisms, may be critical for identifying and prioritizing $\mathrm{AD}$ therapeutic strategies (9). Molecular networks identified in AD studies that demonstrate overlap with those elucidated in aging studies might be of particular interest. Given that aging is the most potent risk factor for sporadic AD, it seems likely that a therapeutic strategy achieving meaningful clinical effects will need to engage aging-relevant mechanisms.

Biologic systems are considered to be highly robust with intrinsic redundant systems and resilience (10). Thus, the existence of $\mathrm{AD}$, and indeed other debilitating and progressive diseases, is likely dependent on the presence of perturbations of multiple fundamental systems. Therapies that do not effectively engage these core mechanisms are unlikely to achieve the clinical effects that patients, families and the field seek. Successful therapeutics will require 'deep biology' approaches, whether achieved by small molecules, biologics, brain stimulation or life-style interventions which fortunately are beginning to appear.

Conflict of interest: Drs. Longo and Massa are listed as inventors on patents relating to AD therapeutics assigned to the University of North Carolina, University of California, San Francisco and the Dept. of Veterans Affairs. Drs. Massa and Longo are entitled to royalties distributed by UC and the VA per their standard agreements. Dr. Longo is a principal of, and has financial interest in PharmatrophiX, a company focused on the development of small molecule ligands for neurotrophin receptors that has licensed several of these patents.

Open Access: This article is distributed under the terms of the Creative Commons Attribution 4.0 International License (http://creativecommons.org/ licenses/by/4.0/), which permits use, duplication, adaptation, distribution and reproduction in any medium or format, as long as you give appropriate credit to the original author(s) and the source, provide a link to the Creative Commons license and indicate if changes were made.

\section{References}

1. Gauthier, S., et al., Non-Amyloid Approaches to Disease Modification for Alzheimer'sDisease: An EU/US CTAD Task Force Report. J Prev Alz Dis 2020;3(7):152-157

2. Aisen, P.S., et al., The Future of Anti-Amyloid Trials 2020. J Prev Alz Dis 2020;3(7):146-151

3. van der Kant, R., L.S.B. Goldstein, and R. Ossenkoppele, Amyloid-betaindependent regulators of tau pathology in Alzheimer disease. Nat Rev Neurosci, 2020. 21(1): p. 21-35.

4. Gallardo, G. and D.M. Holtzman, Amyloid-beta and Tau at the Crossroads of Alzheimer's Disease. Adv Exp Med Biol, 2019. 1184: p. 187-203.

5. Canchi, S., et al., Integrating Gene and Protein Expression Reveals Perturbed Functional Networks in Alzheimer's Disease. Cell Rep, 2019. 28(4): p. 1103 $1116 \mathrm{e} 4$.

6. Seyfried, N.T., et al., A Multi-network Approach Identifies Protein-Specific Co-expression in Asymptomatic and Symptomatic Alzheimer's Disease. Cell Syst, 2017. 4(1): p. 60-72 e4.

7. Zhang, Q., et al., Integrated proteomics and network analysis identifies protein hubs and network alterations in Alzheimer's disease. Acta Neuropathol Commun, 2018. 6(1): p. 19.

8. Colom-Cadena, M., et al., The clinical promise of biomarkers of synapse damage or loss in Alzheimer's disease. Alzheimers Res Ther, 2020. 12(1): p. 21.

9. Mostafavi, S., et al., A molecular network of the aging human brain provides insights into the pathology and cognitive decline of Alzheimer's disease. Nat Neurosci, 2018. 21(6): p. 811-819.

10. Zitnik, M., et al., Evolution of resilience in protein interactomes across the tree of life. Proc Natl Acad Sci U S A, 2019. 116(10): p. 4426-4433. 